\title{
Development of an LC-MS/MS method for the analysis of serotonin and related compounds in urine and the identification of a potential biomarker for attention deficit hyperactivity/hyperkinetic disorder
}

\author{
Merisa Moriarty • Aoife Lee • Brendan O'Connell • \\ Ann Kelleher • Helen Keeley • Ambrose Furey
}

Received: 12 May 2011 /Revised: 6 July 2011 / Accepted: 6 August 2011

(C) Springer-Verlag 2011

\begin{abstract}
Serotonin is a major neurotransmitter and affects various functions both in the brain and in the rest of the body. It has been demonstrated that altered serotinergic function is implicated in various psychiatric disorders including depression and schizophrenia. Serotonin has also been implicated along with dopamine in attention deficithyperkinetic disorder (AD-HKD). This study provides a versatile validated method for the analysis of serotonin, hydroxyindole acetic acid and dopamine in urine using LCMS/MS. This method was then used to quantify these analytes in a test group of 17 children diagnosed with severe AD-HKD. This group was compared to a matched control group to investigate the possibility that one of these compounds may be a potential biomarker for this condition. The developed method provided good linear calibration curves for the multiplex assay of analytes in urine (0.05-
\end{abstract}

Electronic supplementary material The online version of this article (doi:10.1007/s00216-011-5322-7) contains supplementary material, which is available to authorized users.

M. Moriarty $\cdot$ A. Furey $(\bowtie)$

Team Elucidate, Department of Chemistry,

Cork Institute of Technology,

Cork, Ireland

e-mail: Ambrose.Furey@cit.ie

\section{A. Lee $\cdot$ B. O'Connell}

Department of Biological Sciences, Cork Institute of Technology,

Bishopstown,

Cork, Ireland

\section{A. Kelleher · H. Keeley}

Child and Adolescent Mental Health Services,

Health Service Executive, South, North Cork Area

and the National Suicide Research Foundation,

Cork, Ireland $\left.3.27 \mathrm{nmol} / \mathrm{L} ; R^{2} \geq 0.9977\right)$. Acceptable inter-day repeatability was achieved for all analytes with RSD values $(n=9)$ ranging from $1.1 \%$ to $9.3 \%$ over a concentration range of $0.11-3.27 \mu \mathrm{mol} / \mathrm{L}$ in urine. Excellent limits of detection (LOD) and limits of quantitation (LOQ) were achieved with LODs of $8.8-18.2 \mathrm{nmol} / \mathrm{L}$ and the LOQs of 29.4 $55.7 \mathrm{nmol} / \mathrm{L}$ for analytes in urine. Recoveries were in the ranges of $98-104 \%, 100-106 \%$ and $91-107 \%$ for serotonin, 5-HIAA and dopamine, respectively. An appropriate sample clean-up procedure for urine was developed to ensure efficient recovery and reproducibility on analysis. Evaluation of matrix effects was also carried out and the influence of ion suppression on analytical results reported. Confirmatory analysis was carried out on a linear trap quadrupole-Orbitrap mass spectrometer to obtain high mass accuracy data of the target analytes in the clinical samples.

Keywords Attention deficit-hyperactivity/hyperkinetic disorder · AD-HKD · LC-MS/MS · Serotonin · Dopamine . Hydroxyindole acetic acid - Urine

\section{Introduction}

Serotonin (5-hydroxytryptamine, 5-HT) is a tryptophanderived biogenic amine that acts as a neurotransmitter in the central and peripheral nervous systems in the body [1]. It controls many brain functions, regulates blood pressure and smooth muscle contraction. It has been strongly implicated in several pathological conditions such as hypertension, migraine, depression, schizophrenia, anxiety, anorexia nervosa, dementia and carcinoid syndrome. In addition, it has been associated with sleep disruption, aggression, 
temperature regulation, sexual behaviour, and pain sensation [1-6]. It has also been linked to Alzheimer's disease and vascular dementia along with other neurotransmitters such as dopamine [7].

Serotonin is formed by decarboxylation of 5hydroxytryptophan in one of tryptophan's metabolic pathways. Figure 1 shows the synthesis of serotonin from tryptophan and its metabolism into melatonin, 5hydroxyindole acetic acid (HIAA) and hydroxytryptophol. Serotonin undergoes oxidative deamination followed by subsequent oxidation to form 5-HIAA, which is the predominant metabolite in urine [8]. The amount of serotonin, its synthesis, uptake sites, and receptor binding are all higher in the developing brain, compared with adult values, and these levels decline before puberty. This developmental process has been reported to be disrupted in autistic children [1].

Dopamine is also a neurotransmitter but has its own system of neurons. It has been shown that serotonin can influence the dopaminergic system [9]. The dopaminergic system is thought to affect a wide range of behaviours and functions, including cognition, motor function, brain-stimulation reward mechanisms, eating and drinking behaviours, sexual behaviour, neuroendocrine regulation and selective attention [10].

Attention deficit-hyperactivity disorder (AD-HKD) is a highly heritable condition which starts in early childhood and has a large impact on a person's socialisation in later years [11]. Children with AD-HKD disorder experience serious social, academic and psychological impairment. The key symptoms of the condition include inattention, motor hyperactivity and impulsiveness [12]. This condition is one of the most prevalent childhood psychiatric disorders affecting $3-5 \%$ of school-aged children and may it progress into adulthood [13]. All clinical samples were taken under the medical supervision of co-author $\mathrm{Dr} H$. Keeley, Consultant Child and Adolescent Psychiatrist.

Disruption of normal monaminergic neurotransmission has been seen as the crucial mechanism linked with AD-HKD [12]. Although many studies have shown that dopamine plays an important role [14], other monoamines like noradrenaline and serotonin have also been linked to this condition [15]. In fact, while most therapeutic agents used in the treatment of $\mathrm{AD}-\mathrm{HKD}$ are dopaminergic in action, many of the major clinical indicators, e.g. impulsivity are considered to be related to serotinergic function. Also, tricyclic antidepressants and other antidepressants which block the uptake of serotonin have shown to reduce AD-HKD symptoms [16]. Spivak et al. reported that a chronic deficit of serotonin may contribute to the clinical symptoms of the disorder $[17,18]$.

The difficulty with AD-HKD diagnosis is that there is no specific clinical feature or testing procedure and diagnosis is based on clinical assessment of behaviour along with psychological and neurocognitive evaluation [19]. These evaluations are usually based on a structured interview together with symptoms rated by parents and teachers in different settings using diagnostic scales [20]. The major concerns here are that there may be some children that go undiagnosed and who without intervention risk dysfunction, disability or social impairment and others that may be medicated with psychostimulants without need and suffer the harsh side effects of these drugs such as insomnia, appetite suppression and dysphoria [16].
Fig. 1 Synthesis and metabolism of serotonin

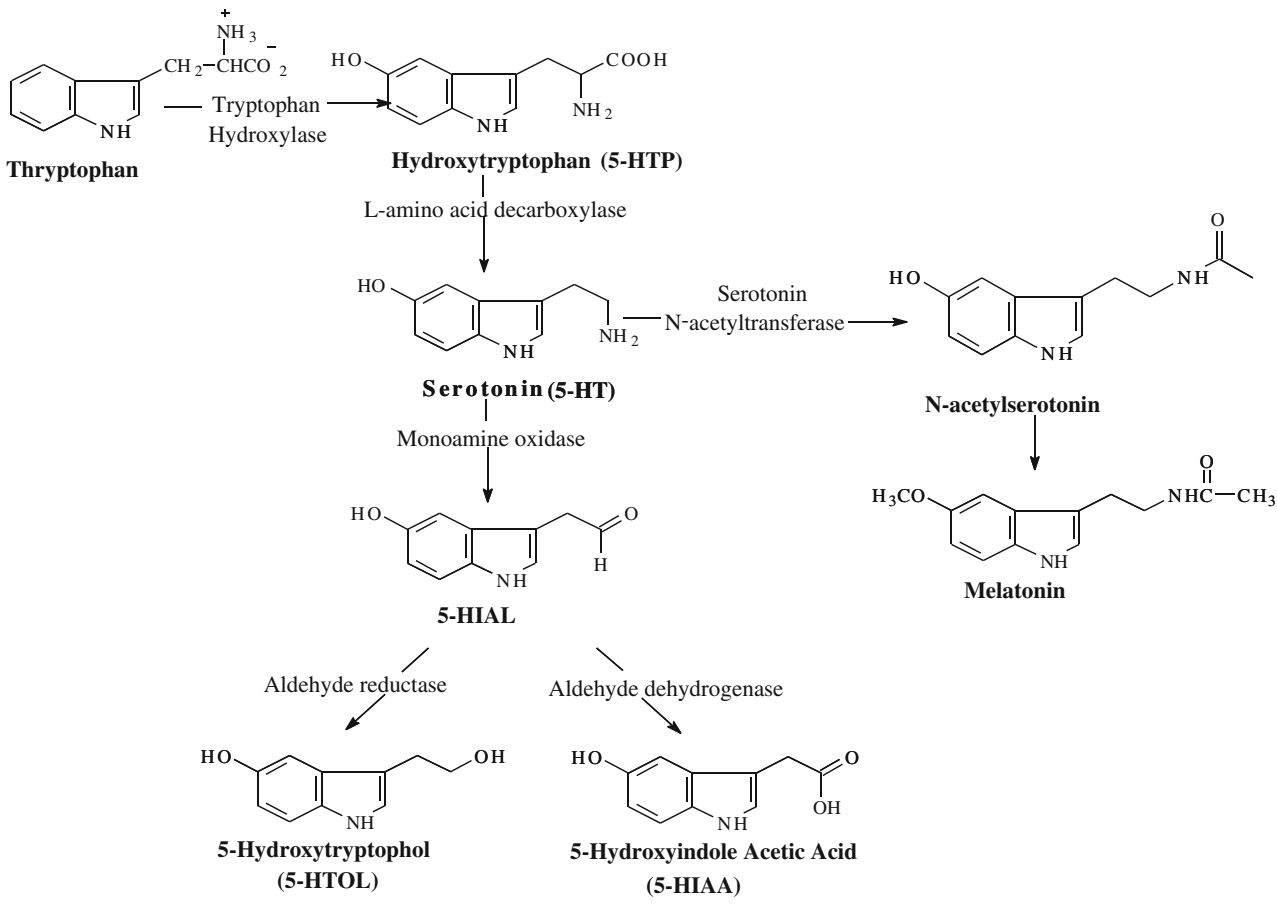


The aim of this study was to identify possible peripheral biological markers for $\mathrm{AD}-\mathrm{HKD}$ in order to aid with diagnosis and to monitor the efficacy of treatment. This required the development of an accurate and robust method for the analysis of the possible biomarkers.

There are several methods published on the analysis of serotonin and other related compounds in biological fluids. A variety of methods have been developed to measure serotonin including spectrophotometry [21, 22], LCfluorometry [23, 24], enzyme immunoassay, and LCelectrochemical detection $[25,26]$. LC-mass spectrometric methods is however the method of choice as tandem LCMS methods provide increased selectivity while reducing the need for dependence on chromatographic separation [27-35]. While these methods report good results for the analytes under investigation, the methods do not facilitate multi-target analysis which was the aim of this study. Extensive method optimisation was required in order to obtain optimum results for the simultaneous analysis of all the pertinent analytes. The LC-MS/MS based method described herein delivers excellent sensitivity, selectivity and robustness for dopamine, serotonin and its key metabolite, hydroxyindole acetic acid in clinical samples.

\section{Materials and method}

\section{Materials}

Chemicals used in this study included formic acid purchased from Merck (Darmstadt, Germany); ammonium acetate and acetic acid were purchased from SigmaAldrich (Dublin). Hydrochloric acid and all HPLC-grade solvents (methanol, water, acetonitrile) were purchased from Labscan (Dublin, Ireland). Nitrogen gas was purchased from Irish Oxygen (Ireland). All standards were purchased from Sigma-Aldrich (Dublin, Ireland) and deuterated compounds were purchased from CDN Isotopes (Quebec, Canada). Isolute C18 cartridges were purchased from Biotage (Sweden). Following ethical approval from the Clinical Ethics Research Committee of the Cork Teaching Hospitals samples were received from a test group of 17 children with a clinical diagnosis of AD-HKD attending a Child and Adolescent Mental Health Service, as well as a matched control group.

\section{Standard preparation}

Standards (serotonin, dopamine and 5-HIAA) in solvent (water) were prepared from a stock mixture of all compounds $(10 \mu \mathrm{g} / \mathrm{ml})$ by serial dilutions. The deuterated internal standards were subsequently added with final internal standard concentrations for serotonin- $d_{4}$ and hydroxyindole acetic acid- $d_{2}$ of 1.11 and $2.07 \mu \mathrm{mol} / \mathrm{L}$, respectively. A deuterated internal standard was purchased for dopamine but it was found that an impurity in the mobile phase had an identical mass $(m / z 158)$ to the deuterated standard which caused continuous interference in the chromatographic run. This resulted in the deuterated dopamine standard being unsuitable as an internal standard. For the urine validation study, aliquots of stock standard solutions were spiked into water that was $\mathrm{pH}$ matched with urine.

\section{Sample preparation}

On collection of urine samples $(10-40 \mathrm{ml}), 1 \mathrm{M} \mathrm{HCl}(0.5 \mathrm{ml})$ was added as a preservative and samples were extracted and analysed within $24 \mathrm{~h}$ of collection, the remainder of the samples were stored at $-24^{\circ} \mathrm{C}$. Samples were centrifuged $(3,000 \times g)$ and the internal standards (serotonin- $d_{4}$ and 5HIAA- $d_{2}, 200 \mu \mathrm{l}$ ) were applied to the urine samples $(200 \mu \mathrm{l})$. Water acidified to $\mathrm{pH} 3.5$ with acetic acid $(800 \mu \mathrm{l})$ was applied and the samples were then vortexed. The SPE procedure contained the following steps: the Isolute C18 cartridges were conditioned with methanol $(3 \mathrm{ml})$, followed by water $(\mathrm{pH} 3.5,3 \mathrm{ml})$, the sample containing water $(1,000 \mu \mathrm{l})$ was then loaded onto the cartridge, washed with a methanol solution $(5 \%, 1 \mathrm{ml})$ and then eluted with methanol with $0.1 \mathrm{M}$ ammonium acetate $(5 \mathrm{ml})$. Samples were then dried under nitrogen and reconstituted in water $(200 \mu \mathrm{l})$. An aliquot $(5 \mu \mathrm{l})$ was injected into the LC-MS/MS.

Separately, quantitation of creatinine in the urine samples were carried out on an Olympus AU640 analyser in an accredited laboratory at Cork University Hospital, the assay principle used for creatinine method was the Jaffe method.

Study of the stability of serotonin, dopamine and hydroxyindole acetic acid in urine

A urine sample $(10 \mathrm{ml})$ was collected and divided into three groups, each group containing two $1 \mathrm{ml}$ aliquots, one of which was acidified with $\mathrm{HCl}$ as a preservative and the other left unaltered. Group 1 was stored at room temperature $\left(18^{\circ} \mathrm{C}\right)$, group 2 was stored at $4^{\circ} \mathrm{C}$ and group 3 was stored at $-24^{\circ} \mathrm{C}$ for four consecutive days. Aliquots $(200 \mu \mathrm{l})$ of all six samples were prepared as in Section Sample Preparation and analysed on each of the 4 days to assess the stability of serotonin, dopamine and hydroxyindole acetic acid.

Instrumentation and Analytical Conditions

Liquid chromatography-tandem mass spectrometry (LC-MS/MS)

A liquid chromatography-tandem mass spectrometry (LCMS/MS) method was developed for the quantification of 
serotonin, dopamine, and 5-HIAA in urine. In order to ensure unambiguous identification, more than one multiple reaction monitoring (MRM) experiment was conducted on each peak, i.e. three product ions (Q3) were selected for each precursor ion (Q1). Separation was performed on an Agilent 1100 series HPLC system (Agilent, Palo Alto, CA, USA) coupled to an API 3000 (Applied Biosystems, Warrington, UK) triple quadrupole mass spectrometer equipped with a turbo-assisted ionspray (ESI) ionisation source (Sciex, Toronto, Canada) in positive mode. Analyst 1.5 software was used for instrument control, data acquisition and data analysis. The sample vials were maintained at $4{ }^{\circ} \mathrm{C}$ in the thermostatic autosampler. Chromatographic separation was obtained using gradient elution on a reverse phase Hypersil Gold aQ column, $3 \mu \mathrm{m}, 150 \times$ $2.1 \mathrm{~mm}$ (Thermo) at $20^{\circ} \mathrm{C}$ at a flow rate of $200 \mu \mathrm{l} / \mathrm{min}$. Separation was performed using a binary gradient composed of mobile phase A, $0.1 \%$ formic acid in water and mobile phase $\mathrm{B}, 0.1 \%$ formic acid in $\mathrm{MeOH}$. A ramped gradient profile was used as follows: (a) $0 \min 90 \% \mathrm{~A}$; (b) $3 \min 80 \%$ A; (c) $3.01 \min 20 \% \mathrm{~A}$; (d) $5.5 \min 50 \%$ A (e) $7 \rightarrow 12 \mathrm{~min} 20 \% \mathrm{~A}$; (f) $12.5 \rightarrow 20 \mathrm{~min} 90 \%$ A. The injection volume was $5 \mu \mathrm{l}$ and the eluent flow was diverted to waste for $2 \mathrm{~min}$ after sample injection and again after $13 \mathrm{~min}$. MS detection was carried out between 1 and $15 \mathrm{~min}$ of the chromatography, total run time of the analysis was $20 \mathrm{~min}$; the dwell time of the method was $1.82 \mathrm{~min}$. The mass spectrometer was operated in positive mode. Q1/Q3 ion pairs outlined in Table 1 were used to allow simultaneous detection of serotonin and related compounds. Optimised values of the MS instrument along with observed ion ratios can be seen in Table 1. Other parameters were kept constant for all analytes, these were, nebuliser gas 8, curtain gas 12 , CAD gas 5 , ion source voltage $5,000 \mathrm{~V}$, ion source temp $370^{\circ} \mathrm{C}$. Once every 3 months, the instrument was calibrated following manufacturer standard operating procedures; however, daily controls and calibration checks were performed for the target compounds to ensure detector stability. No deviation outside $\pm 2.5 \%$ were observed during this investigation.

\section{Liquid chromatography-tandem mass spectrometry (LC-FT-MS)}

Liquid chromatography coupled to a linear trap quadrupoleOrbitrap mass spectrometer (LTQ-Orbitrap, Thermo, USA) was used to obtain high mass accuracy data of the analytes in urine samples. Samples were analysed at 100,000 resolution and the accurate mass of each analyte was determined to four decimal places. The instrument was operated in full scan mode and ion source parameters were optimised for the analytes. H-ESI source parameters were spray voltage $3 \mathrm{kV}$, sheath gas 84 , auxiliary gas 11 , vaporiser temperature $300^{\circ} \mathrm{C}$, capillary temperature $275^{\circ} \mathrm{C}$, capillary voltage $6 \mathrm{~V}$ and tube lens $3 \mathrm{kV}$. The same column and mobile phase gradient was used as in the section "Liquid chromatography-tandem mass spectrometry (LC-MS/MS)".

\section{Ion suppression post column infusion assessment}

Post column infusion experiments were conducted to determine ion suppression/enhancement effects using the approach described by Choi et al. [36]. The experimental setup used in these experiments involved the infusion of a standard during the analysis of a blank matrix solution. A standard mixture $(2.8,2.6$ and $3.3 \mu \mathrm{mol} / \mathrm{L}$ for serotonin, 5HIAA and dopamine, respectively), monitored by MRM, was introduced at $5 \mu \mathrm{l} / \mathrm{min}$ into the LC eluent post-column. A blank matrix (LC-grade water) was injected $(5 \mu \mathrm{l})$ via the autosampler into the LC system. The response of the standard mixture was monitored continuously to produce a
Table 1 Optimised LC-MS/MS parameters

\begin{tabular}{|c|c|c|c|c|c|c|}
\hline & $\operatorname{Mass}(\mathrm{M}+\mathrm{H})^{+}$ & $\mathrm{DP}(\mathrm{FP})$ & Q1/Q3 masses & Collision energy & Ion ratio & $\mathrm{SD}$ \\
\hline \multirow[t]{3}{*}{ Serotonin } & 177 & 28 & $177 / 160$ & 18 & 1 & \\
\hline & & (93) & $177 / 132$ & 30 & 0.14 & 0.009 \\
\hline & & & $177 / 115$ & 40 & 0.17 & 0.014 \\
\hline \multirow[t]{3}{*}{ Serotonin- $\mathrm{d}_{4}$} & 181 & 26 & $181 / 164$ & 18 & 1 & \\
\hline & & (94) & $181 / 136$ & 35 & 0.12 & 0.012 \\
\hline & & & $181 / 119$ & 40 & 0.08 & 0.008 \\
\hline \multirow[t]{2}{*}{ 5-HIAA } & 192 & 41 & $192 / 146$ & 25 & 1 & \\
\hline & & (109) & $192 / 119$ & 45 & 0.08 & 0.011 \\
\hline \multirow[t]{2}{*}{ 5-HIAA- $\mathrm{d}_{2}$} & 194 & 30 & $194 / 148$ & 23 & 1 & \\
\hline & & $(141)$ & $194 / 120$ & 40 & 0.13 & 0.010 \\
\hline \multirow[t]{3}{*}{ Dopamine } & 154 & 25 & $154 / 137$ & 15 & 1 & \\
\hline & & $(80)$ & $154 / 119$ & 25 & 0.19 & 0.012 \\
\hline & & & $154 / 91$ & 35 & 0.47 & 0.012 \\
\hline
\end{tabular}


profile of the matrix effect. Any drop in the constant baseline signal ('suppression of signal') indicates presence of interfering matrix components [37-39]. The process was repeated for urine samples. Five different urine samples were tested to access any possibility for sample-specific ion suppression effects.

\section{Validation}

Validation of the method was carried out in compliance with the EC [40] and ICH [41] guidelines.

Specificity This method utilises retention time stability, chromatographic separation and ion ratio to determine specificity.

Linearity The linear range of this method was investigated for serotonin, 5-HIAA and dopamine in urine by construction of a linear curve by serial dilution of analyte compounds. The range of the analytical method was established by confirming an acceptable degree of linearity, trueness and precision within the specified range.

Limits of detection and quantitation The limit of detection (LOD) and the limit of quantitation (LOQ) were evaluated from the signal to noise ratio $(S / N)$. The LOD and LOQ were calculated for each compound in urine. The $S / N$ was established from the standard deviation of the Yintercept in six low range calibrations which is then divided by the slope of the average calibration for the six calibrations. This value was then multiplied by 3.3 for the LOD and 10 for the LOQ.

Trueness The trueness of this method was expressed as percentage relative error of three different concentrations spiked into blank urine, these concentrations were 2,837, 1,135 and $284 \mathrm{nmol} / \mathrm{L}$ for serotonin, 2,618, 1,046 and $262 \mathrm{nmol} / \mathrm{L}$ for 5 -HIAA and 3,268, 1,306 and $326 \mathrm{nmol} / \mathrm{L}$ for dopamine. The trueness compares the true value and the value obtained by analysis. Recovery experiments were also carried out, where known concentrations of standard were spiked into urine matrix.

Precision The precision of the method was evaluated using both intra-day and inter-day studies. Sample numbers of six and nine were used for intra-day repeatability and inter-day reproducibility respectively. Precision was studied using six concentrations over the linear ranges,

Application of mass spectrometry method for serotonin, dopamine and hydroxyindole acetic acid in urine

Urine samples of 20 control samples and 17 test samples were analysed using the validated method. The urine sample concentrations were expressed as a ratio of creatinine concentration in each sample to correct for possible difference in kidney function. This is required to allow accurate quantitation from a single urine sample as $24 \mathrm{~h}$ sample collection was not feasible. The presence of the analytes was confirmed using high-resolution mass spectrometry on a LTQ-Orbitrap mass spectrometer.

\section{Results and Discussion}

The purpose of this paper is to describe a method that was developed for the analysis of serotonin, 5-HIAA and dopamine in urine samples in order to determine if any of these analytes may be a possible biomarker for AD-HKD in children. There have been a variety of LC-MS methods developed for these compounds but none that were as sensitive, selective and robust as the method presented here.

Other methods of interest in recent years include: Monaghan et al. [42] provide a method for the analysis of serotonin in platelet depleted plasma for use as a biomarker in conditions such as functional gastrointestinal disorders. Protein precipitation is used for sample preparation followed by a cation exchange on-line clean-up step. The LC-MS/MS method has a 6 min analysis, with good recovery $(98 \%$ average over range $89.5-115.5 \mathrm{nmol} / \mathrm{L}$ ) and sensitivity (LOD and LOQ of 1.5 and $5 \mathrm{nmol} / \mathrm{L}$, respectively). Peterson et al. [43] determined serotonin levels in serum using capillary electrophoresis-TOF-MS. This method has an LOD of 67.3, 6.2 and $27.4 \mathrm{nmol} / \mathrm{L}$ for serotonin, 5-HIAA and dopamine, respectively. Miller et al. [44] report an UPLC-MS method for the analysis of 5-HIAA in plasma as an alternative to urine analysis for the diagnosis of midgut carcinoid tumours. LOD and LOQ for this method were 5 and $15 \mathrm{nmol} / \mathrm{L}$, respectively and the method for this single compound had similar levels of accuracy, precision and recoveries as those reported for this study. While these methods show good sensitivity for the analytes they investigate (serotonin and 5HIAA, respectively), the sample preparation was often laborious or unsuitable for urine samples.

Kushnir et al. [34] and Vuorensola et al. [35] have described LC-MS methods for the analysis of dopamine in urine samples. Kushnir et al. show a similar sensitivity to the method presented here with an LOQ of $16.3 \mu \mathrm{mol} / \mathrm{L}$ ( $29.4 \mu \mathrm{mol} / \mathrm{L}$ presented here). The method has a $3.5 \mathrm{~min}$ run time but suffers from very low extraction recoveries $(71 \% \pm 12 \%)$. The sample preparation is designed specifically for catecholamines and may not be effective for serotonin and 5-HIAA. The method provided by Vuorensola et al. compares multiple methods (LC-EC, CE-UV and CVMS) for the analysis of dopamine and methoxycatecholamines. They conclude that the MS method although more selective is far less sensitive than the CE-UV method. 
Perry and Keevil [33] report an on-line extraction LC-MS method for 5-HIAA in urine. The method has a very short run time and good analyte recovery. However, the sensitivity of the method is extremely low with a LOD and a LOQ of 2.8 and $4.0 \mu \mathrm{mol} / \mathrm{L}$ for 5 -HIAA $(0.010$ and $0.036 \mu \mathrm{mol} / \mathrm{L}$ are reported herein). de Jong et al. [45] developed an on-line SPE LC-MS method for 5-HIAA in urine for diagnosing and monitoring patients with carcinoid tumours. This method has a low sensitivity (LOD and LOQ of 0.10 and $0.13 \mu \mathrm{mol} / \mathrm{L}$ ) and a marginally reduced recovery (81-98\%) in comparison to the method reported here. de Jong et al. [27] have also recently described an automated LC-MS method for serotonin in urinary and plasma samples. The LOQ for serotonin $(30 \mathrm{nmol} / \mathrm{L})$ in urine is matched by this study $(36 \mathrm{nmol} / \mathrm{L})$ and our recoveries are in the range of $88-135 \%$; with absolute recoveries of $83-85 \%$.

\section{Determination of serotonin using LC-MS/MS}

Triple quadrupole mass spectrometry was used to quantify serotonin, dopamine and 5-HIAA in urine. Three Q1/Q3 ion pairs were monitored for each compound. MRM studies were carried out to investigate the fragmentation of the precursor ions into their product ions and establish the collision energy required, so as to provide optimum intensity for each product. This was done by setting up sequences with methods containing various collision energies. For the graphs of these MRM studies see Electronic Supplementary Material Fig. S1. The most abundant MRM ions were chosen for quantitation (as in Electronic Supplementary Material Fig. S1a the 177/160 transition for serotonin was used for quantitation) with additional MRM ion combinations used for unequivocal identification (see Table 1).

The column used in this method was chosen as it gave better retention of early eluting compounds in a high aqueous mobile phase in comparison with other reversed phase columns (C18 and polar-RP columns). The addition of $0.1 \%$ formic acid to the mobile phase gave a good signal response for the analytes and assisted in the retention of dopamine on the LC column so as to ensure that it eluted after the column void volume and also avoided the area of intense ion suppression that occurs in the early chromatography region (see Electronic Supplementary Material Fig. S2a).

\section{Validation}

\section{Matrix Effects}

Continuous post column infusion of a standard solution ensures that all matrix components in a sample that elute from the column are ionised along with the analyte [46]. This allows areas of ion suppression or enhancement to be easily identified. The profile for the urine samples (see Electronic Supplementary Material Fig. S2) display areas of both ion suppression and enhancement particularly close to the elution time of dopamine but through manipulation of the chromatographic gradient the dopamine peak was optimised to elute in an ion suppression free region (Electronic Supplementary Material Fig. S2a). Both serotonin and 5-HIAA are relatively unaffected by matrix effects (Fig. S2b and c, Electronic Supplementary Material) following assessment with different urine samples $(n=5)$.

A standard addition experiment was carried out on the urine samples (see Electronic Supplementary Material Fig. S3) and the values obtained were compared to those achieved for the optimised simple calibration method; see Table 2. A large deviation between the two would indicate the presence of significant ion suppression. The standard addition samples correlate well with the simple calibration method with results showing insignificant differences for all analytes (an error of $3.12 \%, 6.13 \%$ and $6.07 \%$ for serotonin, 5-HIAA and dopamine respectively were observed; Electronic Supplementary Material Fig. S3a-c).

\section{Specificity}

Retention time stability of an analyte is an important parameter to investigate during validation, as shifting retention times can lead to inaccuracies in identification as well as variations in the signal response of the analyte especially in complex matrices. The high selectivity of MRM in LC-MS/MS allows for a higher degree of confidence in using retention time as identification marker. The EC council directive 2002/657/EC (implementing the 96/23) [40] suggests that retention time should not vary more than 5\%. For this method retention times were recorded over 3 days with three repeat injections per day of seven standards in the range of $(105-3,268 \mathrm{nmol} / \mathrm{L})$ spiked into blank urine matrix. The retention time varied $\leq 2,2.8$ and $1 \%$ for serotonin, dopamine and 5-HIAA, respectively, this is well below the recommended value of $5 \%$. Analytes with an internal standard showed almost no difference in relative retention time as the deuterated internal references matched the retention characteristics to the analytes of interest.

Table 2 Comparison of the \% error between standard addition results with calibration curve results for analytes in urine matrix

\begin{tabular}{llrrr}
\hline Matrix & Analyte & $\begin{array}{c}\text { Std add } \\
\mathrm{nmol} / \mathrm{L}\end{array}$ & $\begin{array}{l}\text { Cal curve } \\
\mathrm{nmol} / \mathrm{L}\end{array}$ & \% error \\
\hline Urine & Dopamine & 420.4 & 396.3 & 6.1 \\
& Serotonin & 951.1 & 976.1 & 2.6 \\
& 5-HIAA & $8,189.8$ & $7,716.9$ & 6.1 \\
\hline
\end{tabular}




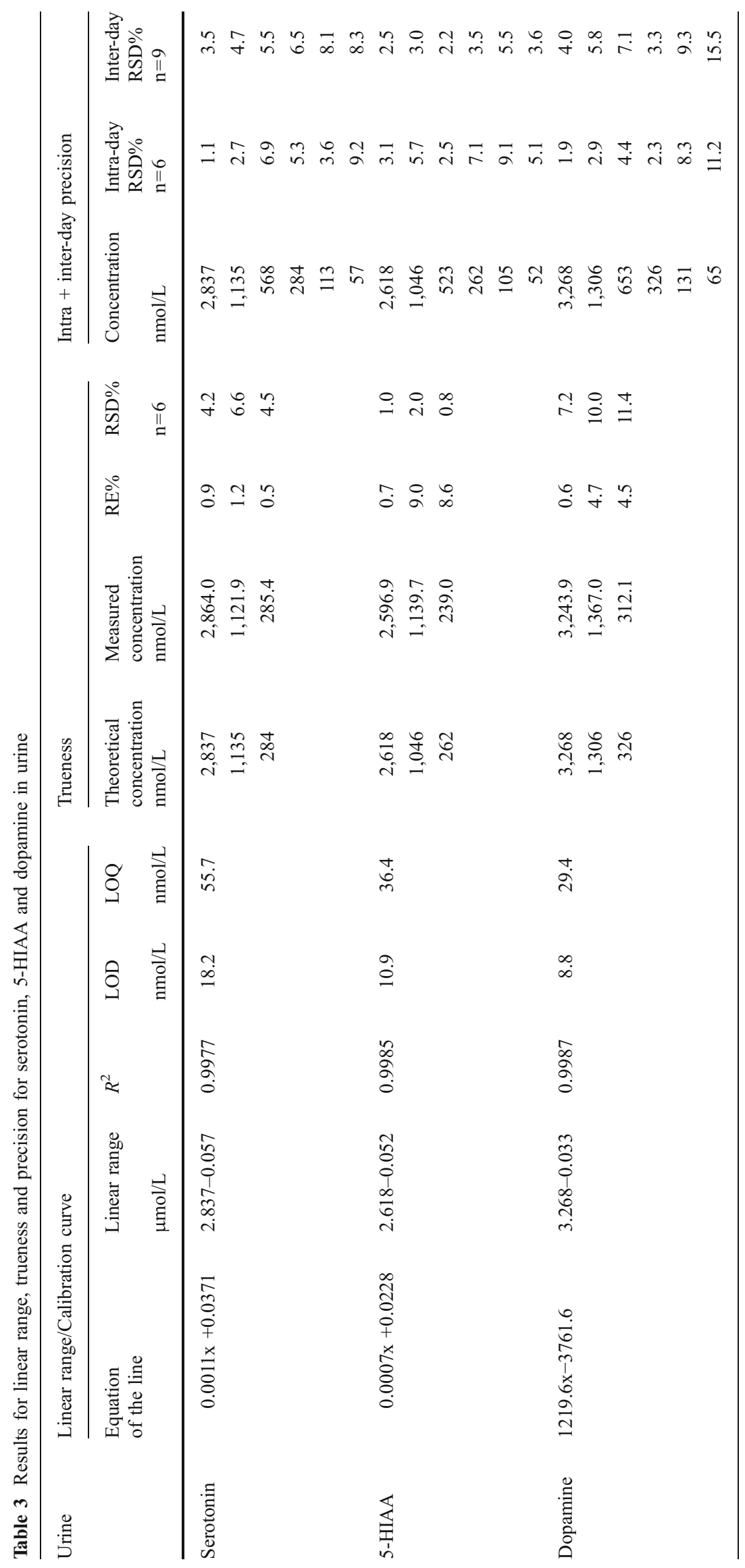


Table 4 Percentage recovery for compounds in urine matrix

\begin{tabular}{lrrrr}
\hline & $\begin{array}{l}\text { Spiked } \\
\text { concentration } \\
\mathrm{nmol} / \mathrm{L}\end{array}$ & $\begin{array}{l}\text { Measured } \\
\text { concentration } \\
\mathrm{nmol} / \mathrm{L}\end{array}$ & Recovery\% & $\begin{array}{l}\text { \% RSD } \\
(\mathrm{n}=5)\end{array}$ \\
\hline Urine & & & & \\
Serotonin & 284 & 278.6 & 98.1 & 4.3 \\
& 1,135 & $1,174.8$ & 104.1 & 2.5 \\
5-HIAA & 262 & 263.6 & 100.8 & 7.6 \\
& 1,046 & $1,108.3$ & 106.0 & 4.0 \\
Dopamine & 326 & 298.3 & 91.3 & 1.2 \\
& 1,306 & $1,398.0$ & 107.2 & 2.0 \\
\hline
\end{tabular}

Possible interferences in the analysis of serotonin are $\mathrm{N}$ acetyl-hydroxytryptamine $(\mathrm{m} / \mathrm{z} 219-160)$ an intermediate in melatonin synthesis and hydroxytryptophol $(\mathrm{m} / \mathrm{z} 178-160)$ mainly found in urine. The gradient elution used in this method ensures that these compounds eluted separately from the serotonin peak (data not shown).

The three most abundant MRM transitions were chosen for each compound, apart from 5-HIAA and 5-HIAA- $d_{2}$ where the two most distinctive MRM ion transitions were employed. Each precursor/product ion allows positive identification of each analyte and the Q1/Q3 ion pairs corresponds to 5.5 identification points according to the $\mathrm{EC}$ directive
2002/657/EC (implementing the 96/23) [40]. The ion ratios for all of the MRM transitions for each of the compounds was monitored over the range of $52-3,268 \mathrm{ng} / \mathrm{ml}$. The ion ratios and their standard deviations over this range are provided in Table 1 . The ion ratios seen here were observed to be common for all calibrations in standards and samples, indicating that they can be used for analyte confirmation.

\section{Linearity}

The linear range was between 0.033 and $3.268 \mu \mathrm{mol} / \mathrm{L}$ for dopamine, 0.057 and $2.84 \mu \mathrm{mol} / \mathrm{L}$ for serotonin and 0.053 and $2.618 \mu \mathrm{mol} / \mathrm{L}$ for 5 -HIAA. Table 3 shows the linear range, equation of line and correlation coefficient for each compound in urine. This method showed good linearity with correlation coefficients $\geq 0.9977$ for urine $(n=5)$. In the equation of the line presented for each analyte in Table 3, $x$ refers to the concentration of the analyte for serotonin, 5HIAA and dopamine in $\mathrm{nmol} / \mathrm{L}$. The $y$ refers to the ratio of peak area of analyte to peak area of internal standard for serotonin and 5-HIAA and peak area alone for dopamine.

\section{Limits of detection and quantitation}

Table 3 shows LOD and LOQ values for serotonin, 5-HIAA and dopamine in urine matrices. The LODs and LOQs for a

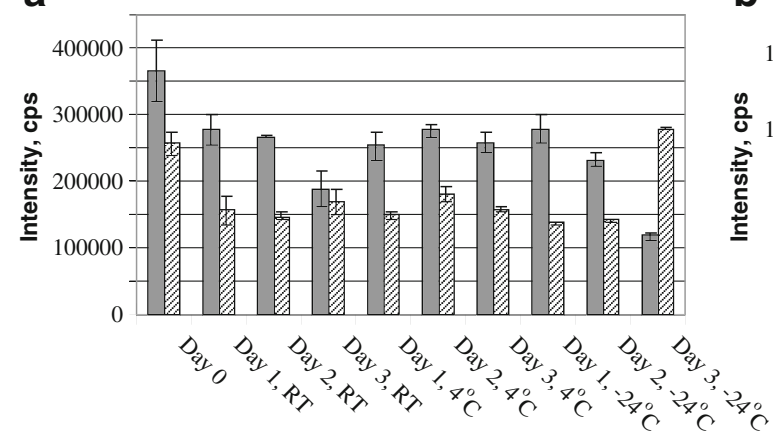

C

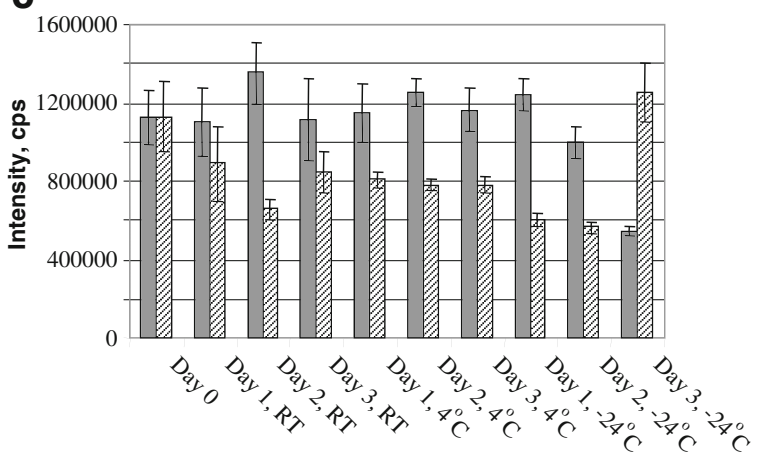

b

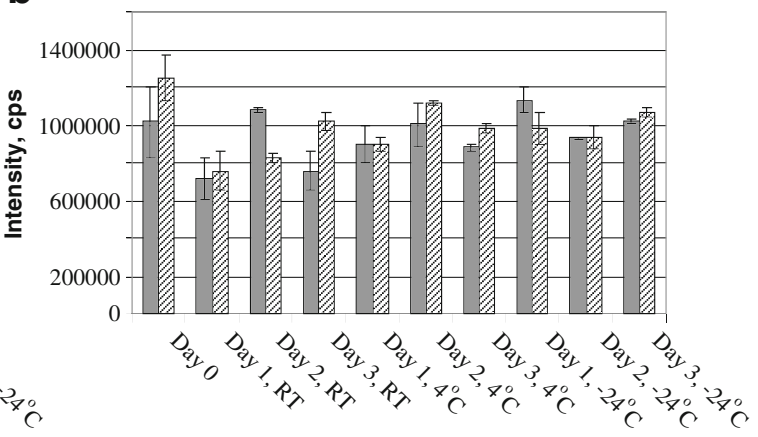

Without Preservative

With Preservative

Fig. 2 Stability study of serotonin (a), dopamine (b) and 5-HIAA (c) in urine over 4 days at room temperature (RT), $4^{\circ} \mathrm{C}$ and $-24^{\circ} \mathrm{C}$ with and without an acid preservative 
Fig. 3 a Chromatogram of dopamine (1), serotonin (2), and 5-HIAA (4), in solvent, with $1.3,1.1$ and $1.0 \mu \mathrm{mol} / \mathrm{L}$, respectively of each standard and $1.1 \mu \mathrm{mol} / \mathrm{L}$ of serotonin- $d_{4}$ (3) and $2.1 \mu \mathrm{mol} / \mathrm{L} 5$-HIAA- $d_{2}$ (5). (b) and dopamine (1) (2.136e $4 \mathrm{cps})$, serotonin (2) (7.819e $3 \mathrm{cps}$ ), and 5-HIAA (4) $(1.049 \mathrm{e} 5 \mathrm{cps})$ in urine samples. Samples shown were diluted by a factor of 10 , final concentrations were $0.21,1.07$ and $10.52 \mu \mathrm{mol} / \mathrm{L}$, respectively
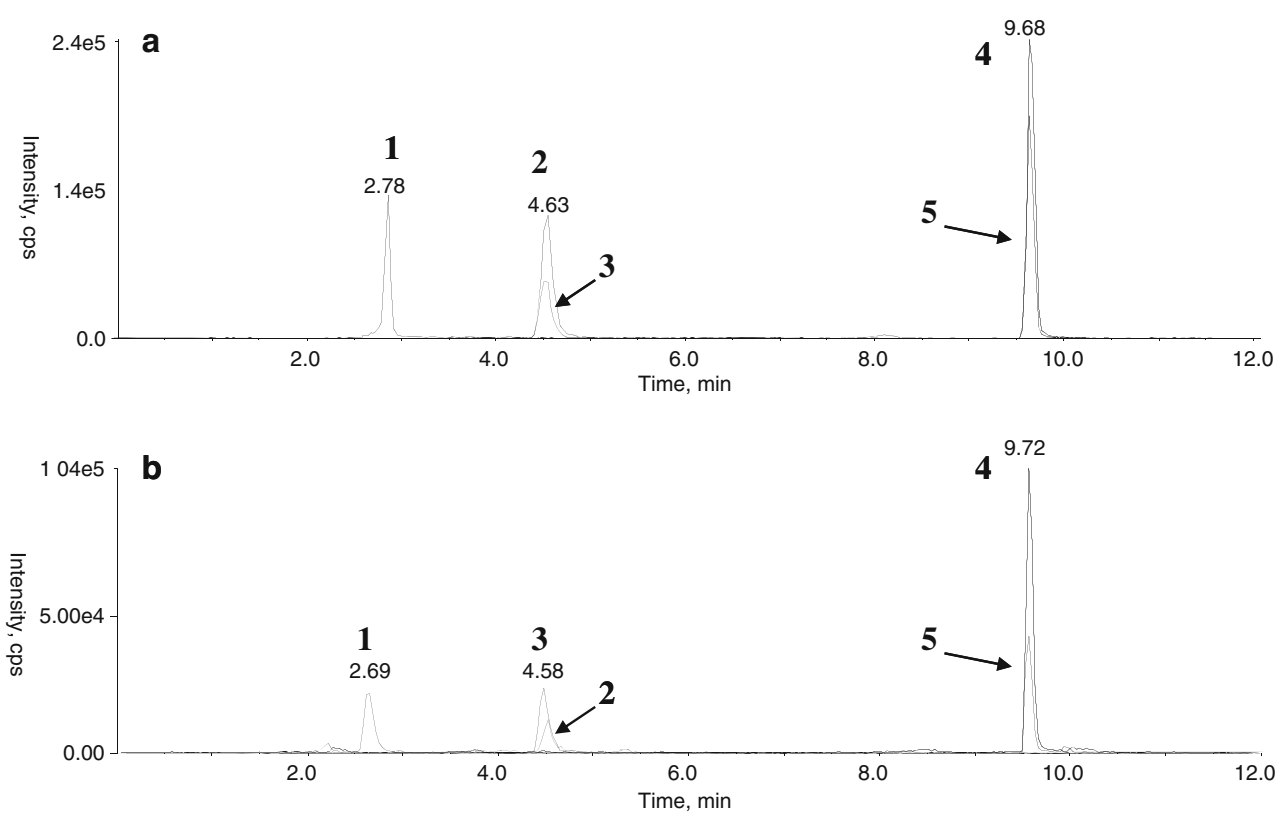

a

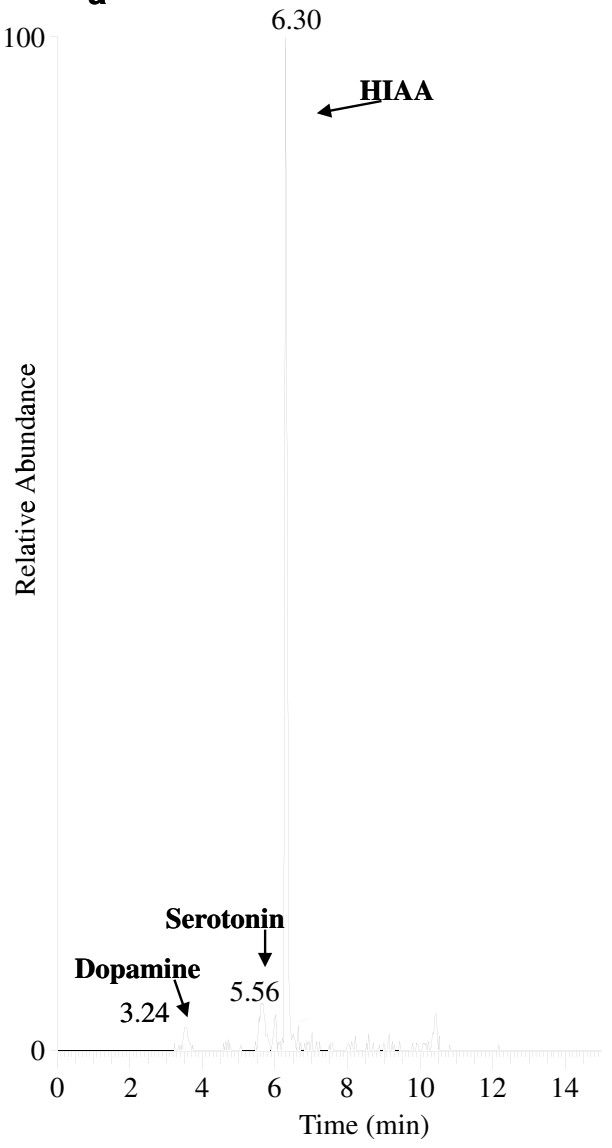

b 100

178.0897
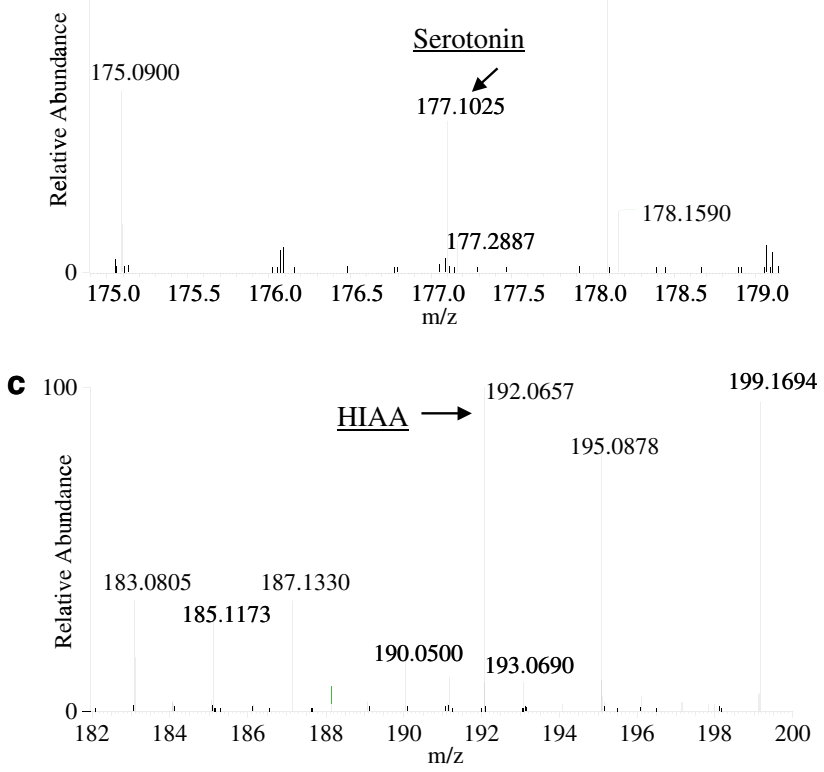

d 100

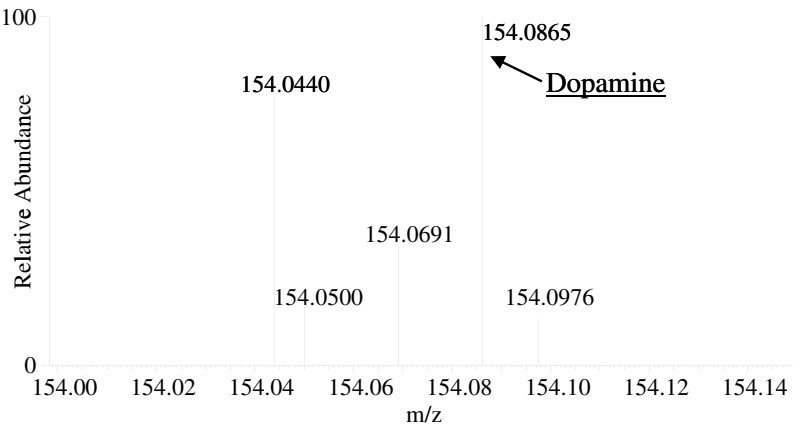

Fig. 4 a High-resolution chromatogram of dopamine, serotonin and 5-HIAA in a urine sample carried out on a LC-LTQ-Orbitrap MS. b-d Spectra of serotonin (PPM error 1.69), 5-HIAA (PPM error 1.56) and dopamine (PPM error 1.95) illustrating high mass accuracy data 
Fig. 5 Diagram showing the interquartile ranges for serotonin (a), 5-HIAA (b) and dopamine (c) concentration ( $\mu \mathrm{mol} / \mathrm{mol}$ creatinine) in test and control urine (a)

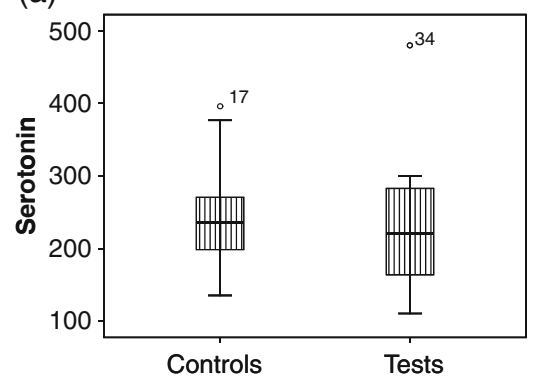

(b)

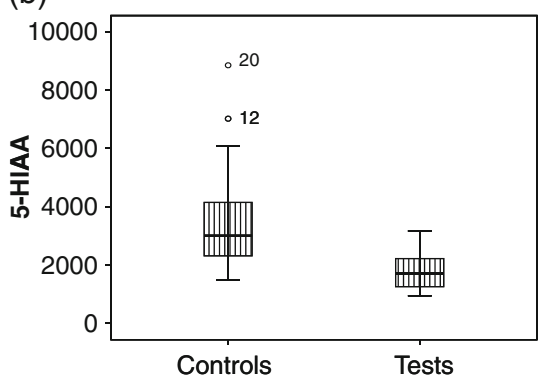

(c)

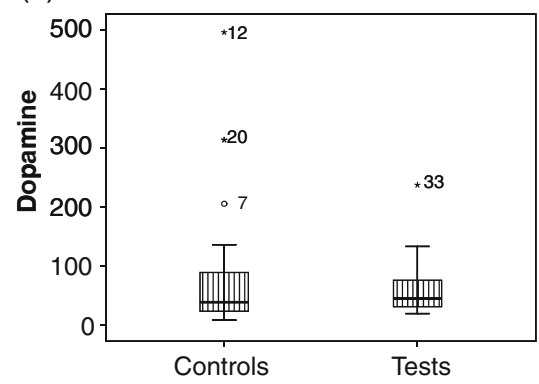

each compound are well below the normal levels usually found in urine samples which are reported as $25-66 \mu \mathrm{mol} /$ $\mathrm{mol}, 0-3.9 \mathrm{mmol} / \mathrm{mol}$ and $28.8-177.8 \mu \mathrm{mol} / \mathrm{mol}$ of creatinine for serotonin, 5-HIAA and dopamine, respectively [47]. In the case of 5-HIAA, the LOD was ca. 1,000 times smaller than normal levels with LODs for serotonin and dopamine being at least ten times lower than normal values. In this method, for the analysis of 5-HIAA in urine the sample is diluted by a factor of ten to allow it to be analysed in the linear calibration range 52-2,618 nmol/L.

\section{Trueness and precision}

The percentage recoveries $(\% \mathrm{RE})$ were very good in the urine matrix giving serotonin values of $\leq 1.16 \%, 5$-HIAA and dopamine gave trueness values of $<9 \%$ and $<5 \%$, respectively, with the higher concentration standard $(2,618$ and $3,268 \mathrm{nmol} / \mathrm{L}$ for 5 -HIAA and dopamine) providing excellent results for both compounds at $\% \mathrm{RE}<1 \%$ (see Table 3).

Recovery experiments were also carried out, these results are summarised in Table 4. These show excellent recoveries for the standards spiked into urine matrix with results of $98-104 \%, 101-106 \%$ and $91-107 \%$ for serotonin, 5-HIAA and dopamine. These results correspond with the post column infusion and standard addition experiments that showed these analytes are relatively unaffected by the ion suppression that is often encountered in the analysis of urine samples.

Precision results were excellent for this method, Table 3 shows a list of the relative standard deviations (RSD) for the concentrations analysed. Percentage RSD values are small (1-6\% for 3,268-523 nmol/L) for all compounds in the urine matrix even at low concentrations $(2-9 \%$ for $326-52 \mathrm{nmol} / \mathrm{L})$.

\section{Sample Stability}

The results of the stability study for serotonin, 5-HIAA and dopamine over four consecutive days can be seen in Fig. 2a-c. Surprisingly, less analyte loss was observed in the unpreserved samples than the acid-preserved samples. It is well known in clinical biochemistry that urine samples for 5-HIAA measurement should be preserved by acidification. However, the stability data in Fig. 2a and b, shows that for serotonin, the signal intensity decreases by approximately $30 \%$ after Day 0 (time zero), indicating that serotonin should ideally be analysed on the day of sample collection. Additionally, Fig. 2a indicates that serotonin appears to be most stable when unpreserved at $4^{\circ} \mathrm{C}$. Regarding 5-HIAA, Fig 2c indicates that, aside from the increase in preserved sample at $-24^{\circ} \mathrm{C}$ at Day 3, 5-HIAA appears most stable when unpreserved at room temperature or at $4^{\circ} \mathrm{C}$. For this study, all samples were extracted and analysed on the day of collection. However, this study

Table 5 Mann-Whitney $U$ statistic and $P$ value

\begin{tabular}{lccc}
\hline & Serotonin & 5-HIAA & Dopamine \\
\hline Mann-Whitney U & 144.00 & 44.00 & 146.00 \\
P value & 0.610 & 0.000 & 0.656 \\
\hline
\end{tabular}

$P$ value $<0.05$ statistically significant

$P$ value $<0.001$ highly statistically significant 
verifies the difficulties that would arise if samples required lengthy storage prior to analysis. This pertinent issue of sample storage will be the focus of future research for the author as samples storage, transportation and extraction is an important consideration in the assessment of AD-HKD patients.

\section{Application of method}

Quantitative measurements were carried out for each of the potential markers in the urine samples that were collected from 17 children who had been clinically diagnosed with AD-HKD. A matched control population was also screened. Calibration standards, blanks and controls were analysed along with the samples in each sequence. Figure 3 shows a chromatogram of serotonin, 5-HIAA and dopamine in a typical standard and urine sample. Urine sample levels for the target analytes for both the control and test groups were expressed as micromoles per mole creatinine to ensure correction for the difference in kidney function (See Electronic Supplementary Material Table S1).

High mass accuracy data was obtained using highresolution settings $(R=100,000)$. The resulting accurate mass values for the target compounds gave low PPM error $(<2 \mathrm{ppm}$ error $)$ for all urine samples. Figure $4 \mathrm{a}$ shows a chromatogram obtained using an LC coupled to an LTQOrbitrap mass spectrometer, this clearly illustrates the presence of three peaks that have accurate masses that correspond to the three analytes under investigation. Figure $4 \mathrm{~b}-\mathrm{d}$ shows the spectra containing the accurate masses of each analyte which are $m / z$ 177.1025, 192.0657 and 154.0865 for serotonin, 5-HIAA and dopamine, respectively. They also illustrate the PPM error associated with the accurate mass measurements. Ions with a mass of $500 \mathrm{Da}$ or less require an accuracy of $0.0025 \mathrm{Da}$ to allow for unambiguous assignment of elemental composition [48]. For serotonin, this corresponds to an error of 14 PPM. As can be seen in Fig. 4b-d, the PPM errors are less than 2 PPM providing results to an accuracy value of within $0.00035 \mathrm{Da}$. These results definitively confirm the presence of these analytes in the urine sample.

The results of analyte concentrations in the test and control groups were compared to assess the possibility of significant differences between the two groups. Figure $5 \mathrm{a}-\mathrm{c}$ shows the interquartile ranges for serotonin, 5-HIAA and dopamine. These diagrams show a distinct difference in 5-HIAA concentrations between the test and control groups, with the test group concentration being considerably lower. Concentrations of serotonin and dopamine showed no obvious difference between the two groups.

The Mann-Whitney $U$ statistic which uses the median of the values provides a much better comparison for the serotonin, 5-HIAA and dopamine levels detected between the Control and Test urines samples. Table 5 provides the Mann-Whitney $U$ statistic and $P$ values for these results. These indicate that for serotonin and dopamine, the difference in concentrations between the control and tests were not significantly different. However, 5-HIAA was significantly lower in the test group in comparison with the control population $(P<0.001)$, a $P$ value less than 0.001 indicates that there is a significant difference between the two populations. Therefore, these results suggest that 5HIAA may be a useful biomarker for AD-HKD.

\section{Conclusion}

This study outlines a validated method for the multiplex assay of serotonin, dopamine and 5-HIAA in urine. The method presented is a robust, sensitive and selective method for the quantitation of these analytes. The method was used to carry out a preliminary study to investigate potential biomarkers for the diagnosis of AD-HKD. From the study, it was concluded that the serotonin metabolite 5HIAA may be a potential biomarker as a significant statistical difference was seen between the control and test groups. Extended studies including larger patient/control cohorts are needed to confirm this hypotheses. The method developed here has the capabilities to be applied to a large cohort study due to its rapidity and robustness with full certainty of analytical data.

Acknowledgments We gratefully acknowledge funding from the Irish Research Council for Science, Engineering and Technology (IRCSET) funding M. Moriarty. The Council of Directors, Technological Sector Research-Strand III 2006 Grant Scheme, awarded to Dr A. Furey is also acknowledged for funding the formation of the 'Team Elucidate' research group. The Higher Education Authority (Programme for Research in Third-Level Institutions, Cycle 4 (PRTLI IV)) National Collaboration Programme on Environment and Climate Changes: Impacts and Responses is also acknowledged. Thanks also to Dr Paul Corcoran for advice regarding statistical analysis.

\section{References}

1. Martínez A, Knappskog PM, Haavik J (2001) A structural approach into human tryptophan hydroxylase and its implications for the regulation of serotonin biosynthesis. Curr Med Chem 8:1077-1091

2. Tsao C-W, Lin Y-S, Chen C-C, Bai C-H, Wu S-R (2006) Cytokines and serotonin transporter in patients with major depression. Prog Neuro-Psychoph 30(5):899-905

3. Ishida J, Takada M, Yamaguchi M (1997) 3,4-Dimethoxybenzylamine as a sensitive pre-column fluorescence derivatization reagent for the determination of serotonin in human platelet-poor plasma. J Chromatogr B 692(1):31-36

4. Nutt D, Argyropoulos S, Hood S, Potokar J (2006) Generalized anxiety disorder: a comorbid disease. Eur Neuropsychopharm 16: S109-S118 
5. Mitani H, Shirayama Y, Yamada T, Kawahara R (2006) Plasma levels of homovanillic acid, 5-hydroxyindoleacetic acid and cortisol, and serotonin turnover in depressed patients. Prog Neuro- Psychoph 30(3):531-534

6. Kema IP, de Vries EGE, Muskiet FAJ (2000) Clinical chemistry of serotonin and metabolites. J Chromatogr-Biomed 747(1-2):33-48

7. Alisky JM (2006) Neurotransmitter depletion may be a cause of dementia pathology rather than an effect. Med Hypotheses 67:556-560

8. Stephanson N, Dahl H, Helander A, Beck O (2005) Determination of urinary 5-hydroxytryptophol glucuronide by liquid chromatography-mass spectrometry. J Chromatogr-Biomed 816 (1-2):107-112

9. Carta M, Fadda F, Stancampiano R (2006) Tryptophan-deficient diet increases the neurochemical and behavioral response to amphetamine. Brain Res 1094(1):86-91

10. Lam KSL, Aman MG, Arnold LE (2006) Neurochemical correlates of autistic disorder: a review of the literature. Res Dev Disabil 27(3):254-289

11. Retz W, Rösler M (2009) The relation of ADHD and violent aggression: what can we learn from epidemiological and genetic studies? Int J Law Psychiat 32(4):235-243

12. Retz W, Thome J, Blocher D, Baader M, Rösler M (2002) Association of attention deficit hyperactivity disorder-related psychopathology and personality traits with the serotonin transporter promoter region polymorphism. Neuroscience Lett 319 (3):133-136

13. Banerjee E, Sinha S, Chatterjee A, Nandagopal K (2009) No causal role for the G482T and G689T polymorphisms in translation regulation of serotonin transporter (SLC6A4) or association with attention-deficit-hyperactivity disorder (ADHD). Neuroscience Lett 454(3):244-248

14. Cook EH, Stein MA, Krasowski MD, Cox NJ, Olkon DM, Kieffer JE, Leventhal BL (1995) Association of attention-deficit disorder and the dopamine transporter gene. Am J Hum Genet 56(4):993998

15. Hawi Z, Dring M, Kirley A, Foley D, Kent L, Craddock N, Asherson P, Curran S, Gould A, Richards S, Lawson D, Pay H, Turic D, Langley K, Owen M, O’Donovan M, Thapar A, Fitzgerald M, Gill M (2002) Serotonergic system and attention deficit hyperactivity disorder (ADHD): a potential susceptibility locus at the 5-HT1B receptor gene in 273 nuclear families from a multi-centre sample. Mol Psychiatr 7(7):718-725

16. Ahmann PA, Theye FW, Berg R, Linquist AJ, Van Erem AJ, Campbell LR (2001) Placebo-controlled evaluation of amphetamine mixture-dextroamphetamine salts and amphetamine salts (Adderall): Efficacy rate and side effects. Pediatrics 107(1)

17. Spivak B, Vered Y, Yoran-Hegesh R, Averbuch E, Mester R, Graf E, Weizman A (1999) Circulatory levels of catecholamines, serotonin and lipids in attention deficit hyperactivity disorder. Acta Psychiatr Scand 99(4):300-304

18. Coleman NS, Foley S, Dunlop SP, Wheatcroft J, Blackshaw E, Perkins AC, Singh G, Marsden CA, Holmes GK, Spiller RC (2006) Abnormalities of serotonin metabolism and its relation to symptoms in untreated celiac disease. Clin Gastroenterol H 4 (7):874-881

19. Davids E, von Bünau U, Specka M, Fischer B, Scherbaum N, Gastpar M (2005) History of attention-deficit hyperactivity disorder symptoms and opioid dependence: a controlled study. Prog Neuro- Psychoph 29(2):291-296

20. Fone KCF, Nutt DJ (2005) Stimulants: use and abuse in the treatment of attention deficit hyperactivity disorder. Curr Opin Pharmacol 5(1):87-93

21. Vinci G, Antonelli ML (2002) Biogenic amines: quality index of freshness in red and white meat. Food Control 13(8):519524
22. Bose D, Durgbanshi A, Capella-Peiro ME, Gil-Agusti A, EsteveRomero J, Carda-Broch S (2004) Micellar liquid chromatography determination of some biogenic amines with electrochemical detection. J Pharm Biomed Anal 36(2):357-363

23. Umeda S, Stagliano GW, Borenstein MR, Raffa RB (2005) A reverse-phase HPLC and fluorescence detection method for measurement of 5-hydroxytryptamine (serotonin) in Planaria. J Pharmzcol Toxicol 51(1):73-76

24. Hara K, Hirowatari Y, Yoshika M, Komiyama Y, Tsuka Y, Takahashi H (2004) The ratio of plasma to whole-blood serotonin may be a novel marker of atherosclerotic cardiovascular disease. $\mathrm{J}$ Lab Clin Med 144(1):31-37

25. Bolandparvaz S, Vasei M, Owji AA, Ata-ee N, Amin A, Daneshbod Y, Hosseini SV (2004) Urinary 5-hydroxy indole acetic acid as a test for early diagnosis of acute appendicitis. Clin Biochem 37(11):985-989

26. Patel BA, Arundell M, Parker KH, Yeoman MS, O’Hare D (2005) Simple and rapid determination of serotonin and catecholamines in biological tissue using high-performance liquid chromatography with electrochemical detection. J Chromatogr B 818(2):269276

27. de Jong WHWM, de Vries EG, Kema IP (2010) Automated mass spectrometric analysis of urinary and plasma serotonin. Anal Bioanal Chem 396(7):2609-2616

28. LA Hammad NM, Bridge B, Mechref Y (2009) Fast liquid chromatography separation and multiple-reaction monitoring mass spectrometric detection of neurotransmitters. J Sep Sci 32 (14):2369-2376

29. El-Beqqali AKA, Abdel-Rehim M (2007) Determination of dopamine and serotonin in human urine samples utilizing microextraction online with liquid chromatography/electrospray tandem mass spectrometry. J Sep Sci 30(3):421-424

30. Numan A, Danielson ND (2002) On-line photo-derivatization with flow injection and liquid chromatography-atmospheric pressure electrospray mass spectrometry for the identification of indoles. Anal Chim Acta 460(1):49-60

31. Hows MEP, Lacroix L, Heidbreder C, Organ AJ, Shah AJ (2004) High-performance liquid chromatography/tandem mass spectrometric assay for the simultaneous measurement of dopamine, norepinephrine, 5-hydroxytryptamine and cocaine in biological samples. J Neurosci Methods 138(1-2):123-132

32. Lang WS, Masucci JA, Caldwell GW, Hageman W, Hall J, Jones WJ, Rafferty BM (2004) Liquid chromatographic and tandem mass spectrometric assay for evaluation of in vivo inhibition of rat brain monoamine oxidases (MAO) $\mathrm{A}$ and $\mathrm{B}$ following a single dose of MAO inhibitors: application of biomarkers in drug discovery. Anal Biochem 333(1):79-87

33. Perry HKB (2008) Online extraction of 5-hydroxyindole acetic acid from urine for analysis by liquid chromatography-tandem mass spectrometry. Ann Clin Biochem 45(Pt 2):149-152

34. Kushnir MMUF, Frank EL, Roberts WL, Shushan B (2002) Analysis of catecholamines in urine by positive-ion electrospray tandem mass spectrometry. Clin Chem 48(2):323-331

35. Vuorensola KSH, Karjalainen U (2003) Determination of dopamine and methoxycatecholamines in patient urine by liquid chromatography with electrochemical detection and by capillary electrophoresis coupled with spectrophotometry and mass spectrometry. J Chromatogr B 788(2):277-289

36. Choi BK, Hercules DM, Gusev AI (2001) LC-MS/MS signal suppression effects in the analysis of pesticides in complex environmental matrices. Fresen J A Chem 369(3-4):370-377

37. Antignac J-P, de Wasch K, Monteau F, De Brabander H, Andre F, Le Bizec B (2005) The ion suppression phenomenon in liquid chromatography-mass spectrometry and its consequences in the field of residue analysis. Anal Chim Acta 529 $(1-2): 129-136$ 
38. Benijts T, Dams R, Lambert W, De Leenheer A (2004) Countering matrix effects in environmental liquid chromatographyelectrospray ionization tandem mass spectrometry water analysis for endocrine disrupting chemicals. J Chromatogr A 1029 (1-2):153-159

39. Kall MA, Fu I, Dige T, Vallano P, Woolf E, Jorgensen M (2007) Development and validation of a selective and sensitive bioanalytical procedure for the quantitative determination of gaboxadol in human plasma employing mixed mode solid phase extraction and hydrophilic interaction liquid chromatography with tandem mass spectroscopic detection. J Chromatogr B 858(12):168-176

40. Commision decision of 12 August 2002 implementing council directive 96/23/EC concerning the performance of analytical methods and the interpretation of results (notified under document number C(2002) 3044) (Text with EEA relevance) $(2002 / 657 / E C)$. L 221/8-36 EN Official Journal of the European Communities 1782002

41. International Conference on Harmonisation of technical requirements for registration of pharmaceutical for human use: Validation of analytical procedures: Text and Methodology Q2(R1) Current Step 4 version, Parent Guideline dated 27 October 1994 (Complementary Guidelines on Methodology dated 6 November 1996 incorporated in November 2005)
42. Monaghan PJ, Brown HA, Houghton LA, Keevil BG (2009) Measurement of serotonin in platelet depleted plasma by liquid chromatography tandem mass spectrometry. J Chromatogr B 877 (22):2163-2167

43. Peterson ZD, Lee ML, Graves SW (2004) Determination of serotonin and its precursors in human plasma by capillary electrophoresis-electrospray ionization-time-of-flight mass spectrometry. J Chromatogr B 810(1):101-110

44. Miller AG, Brown H, Degg T, Allen K, Keevil BG (2010) Measurement of plasma 5-hydroxyindole acetic acid by liquid chromatography tandem mass spectrometry-comparison with HPLC methodology. J Chromatogr B 878(7-8):695-699

45. de Jong WHA, Graham KS, de Vries EGE, Kema IP (2008) Urinary 5-HIAA measurement using automated on-line solidphase extraction-high-performance liquid chromatographytandem mass spectrometry. J Chromatogr B 868(1-2):28-33

46. Choi BK, Hercules DM, Gusev AI (2001) LC-MS/MS signal suppression effects in the analysis of pesticides in complex environmental matrices. Fresen J Anal Chem 369(3-4):370-377

47. Burtis CA, Ashwood ER, Bruns DE Tietz textbook of clinical chemistry and molecular diagnostics. Elsevier Sanders

48. Watson JT, Sparkman OD (2008) Introduction to mass spectrometry: instrumentation, applications and strategies for data intrepretation. 4th edn. Wiley 